\title{
Nasal airflow dynamics: mechanisms and responses associated with an external nasal dilator strip
}

\author{
J.P. Kirkness*, J.R. Wheatley**, T.C. Amis**
}

\begin{abstract}
Nasal airflow dynamics: mechanisms and responses associated with an external nasal dilator strip. J.P. Kirkness, J.R. Wheatley, T.C. Amis. (C)ER Journals Ltd 2000.

ABSTRACT: The adhesive external nasal dilator strip (ENDS) is widely advocated for prevention of snoring and promotion of nasal breathing during exercise. In the present study, the effects of the ENDS on nasal airflow resistance $(R n)$ in normal subjects were examined and factors determining individual responses to the ENDS explored.

Using posterior rhinomanometry, 20 healthy Caucasian adults (10 males, 10 females; age: 18-56 yrs) were studied during quiet tidal breathing and voluntary hyperpnoea with (ENDS) and without (control) ENDS and with a placebo strip (placebo) before and after application of a topical nasal decongestant (oxymetazoline hydrochloride).

During tidal breathing, only nine subjects showed a significantly $(\mathbf{p}<0.05)$ decreased inspiratory and/or expiratory $R \mathrm{n}$ with the ENDS ("responders"). During the control, inspiratory $R \mathrm{n}\left(\right.$ at $\left.0.4 \mathrm{~L} \cdot \mathrm{s}^{-1}\right)$ was higher in "responders" than "nonresponders" $(3.28 \pm$ 0.16 versus $\left.2.60 \pm 0.08 \mathrm{cmH}_{2} \mathrm{O} \cdot \mathrm{L}^{-1} \cdot \mathrm{s} ; \mathrm{p}=0.04\right)$. The effects of nasal decongestant and the ENDS were additive. During voluntary hyperpnoea, inspiratory $R \mathrm{n}\left(\right.$ at $\left.1.0 \mathrm{~L} \cdot \mathrm{s}^{-1}\right)$ and the hysteresis of the inspiratory transnasal pressure/flow curve were decreased with the ENDS in most subjects.

It is concluded that the external nasal dilator strip influences nasal airflow dynamics by both dilation of the nasal valve and stabilization of the lateral nasal vestibule walls and may be more effective in subjects with a high resting nasal airflow resistance.
\end{abstract}

Eur Respir J 2000; 15: 929-936.

The adhesive external nasal dilator strip (ENDS) device (Breathe Right $\mathbb{R}$, CNS, Inc., Minneapolis, MN, USA) offers a simple mechanical approach to the modification of nasal airway resistance $(R \mathrm{n})$. Consisting of two parallel flat polyester springs enclosed by an adhesive tape covering, when properly positioned and fastened to the skin over the dorsum of the nose, this device exerts a truss-like action (resulting from the recoil forces created by bending the polyester springs), lifting the skin (via the adhesive connection) over the lateral nasal vestibule walls. Transmission of this force to the internal nasal walls leads to mechanical dilation of the nasal airway [1].

The site of action of the ENDS is thought to be at the nasal valve and nasal vestibule [1-3]. The nasal valve represents the narrowest cross-sectional area encountered in the normal nasal airway and constitutes a critical flowlimiting segment in the determination of upper airway resistance during nasal breathing $[4,5]$. Consequently, enlargement of the valve area is likely to cause a substantial decrease in $R \mathrm{n}$, especially under high-flow conditions such as may be encountered during exercise.

Recent reports documenting measurements of $R \mathrm{n}$ with ENDSs in normal subjects have produced conflicting results with the ENDS significantly reducing $R \mathrm{n}$ in some studies [2, 6-8] but having no effect in others [9]. In addition, an unexplored feature is the magnitude of the
* School of Science, University of Western Sydney, Nepean, Kingswood, **Dept of Respiratory Medicine, Westmead Hospital and University of Sydney, Westmead, Australia.

Correspondence: J.P. Kirkness, Dept of Respiratory Medicine, Westmead Hospital, Westmead NSW 2145, Australia. Fax: 0061298457286

\section{Keywords: Hyperpnoea}

nasal decongestant

nasal patency

nasal resistance

Received: January 271999

Accepted after revision January 52000

This study was supported by the National Health and Medical Research Council of Australia. between-subject variability in the response to the ENDS, a characteristic likely to have important implications in the practical application of the device to individual subjects. Recently, an almost three-fold intersubject variation in the compliance of the lateral nasal vestibule wall in normal healthy subjects was demonstrated (using the ENDS) [10], and it was suggested that this may explain, at least in part, the variability in responses to ENDSs.

The aim of the present study was to 1) examine the effect of ENDS on $R \mathrm{n}$ in normal healthy subjects under both resting and stimulated breathing conditions, with particular emphasis on the responses of individual subjects; and 2) explore additional factors which may determine the nature of the response to the ENDS in a given individual.

\section{Methods}

Twenty healthy Caucasian adults (10 males, 10 females; age: 18-56 yrs) were studied, none of whom had any symptoms of nasal obstruction at the time of the study. A questionnaire was used to exclude subjects with a history of: 1) allergic rhinitis, 2) recurrent colds or influenza, 3) chronic nasal obstruction, 4) regular use of nasal medications, 5) asthma, 6) obstructive sleep apnoea, 7) snoring more than once per week, or 8) any other nasal complaint. 
Informed consent was obtained from each subject and the protocol was approved by the Western Sydney Area Health Service Ethics Committee.

\section{Nasal airway flow dynamics}

Transnasal pressure/flow relationships were recorded in the upright (seated) position using a modification [11] of conventional posterior rhinomanometry $[12,13]$. Subjects breathed via a nasal continuous positive airway pressure mask (Sullivan; ResMed, Sydney, Australia) connected to a pneumotachograph (Fleisch No. 2, Gould, Bilthoven, the Netherlands or Collins No. 875; Collins Australia, Sydney, Australia) coupled to a differential pressure transducer $\left( \pm 10 \mathrm{cmH}_{2} \mathrm{O}\right.$; Celesco Transducer Products, IDM Instruments, Dandenong, Australia). An occluded mouthpiece (Standard mouthpiece; Sensormedics, Middle Park, Australia) was placed in the subject's mouth and connected to a differential pressure transducer (MP 45; $\pm 100 \mathrm{cmH}_{2} \mathrm{O}$; Validyne, Northridge, CA, USA). The other port of the transducer was connected to the nasal mask. With the occluded mouthpiece in place, there was no oral route airflow and the pressure inside the mouthpiece reflected oropharyngeal pressure. Thus the output of the pressure transducer reflected transnasal pressure. Both flow and pressure signals were digitized $(50-400 \mathrm{~Hz})$, stored on a computer for later analysis and displayed on-line as a transnasal pressure/flow plot. The pressure and airflow signals were in phase to $12 \mathrm{~Hz}$.

\section{Nasal dilator strips}

The adhesive ENDS used in the present study is a commercially available product (Breathe Right $(\mathbb{R}$ ) nasal strips; $3 \mathrm{M}$ Co., Sydney, Australia). The device is available in two different sizes for adults, small/medium and medium/large. All subjects in the present investigation were studied using the small/medium size.

\section{Placebo strip}

Placebo strips (adhesive tape without the polyester springs) were used for comparison with results obtained with ENDSs. In two subjects, the placebo strip was cut to the same shape as the ENDS from tape similar in appearance to that of the ENDS device (Micropore; $3 \mathrm{M} \mathrm{Co}$.). In another 12 subjects, this placebo tape strip was also severed (to prevent development of tension within the strip) across the centre of the strip, whereas six other subjects used placebo strips made by the manufacturer to the same specifications as the ENDS product but without the polyester springs. Placebo strips were presented to the subject in the same packaging as the active devices.

\section{Application of nasal strips}

All ENDSs and placebo strips were placed in accordance with the manufacturer's directions, which specify that the device should be positioned midway over the nose with the tape-covered springs extending down the external lat- eral nasal walls along the nasal crease and with the tabs at each end of the nasal strips adhered to the flare of the nostril. All strips were applied by one operator.

\section{Protocol}

Measurements of nasal airflow dynamics were made, in a randomized, single-blind fashion, for each of the following conditions: 1) with the ENDS, 2) without the ENDS (control), and 3) with the placebo strip (placebo). Subjective assessments of the degree of nasal blockage (nasal patency score (NPS)) were obtained immediately before the transnasal pressure/flow measurements for each condition. Subjects were asked to indicate on a scale of 0 10 their response to the question: "How blocked is your nose?" Studies were performed during both quiet relaxed tidal breathing and voluntary hyperpnoea. During the hyperpnoeic runs, subjects were asked to achieve a target inspiratory airflow of $1 \mathrm{~L} \cdot \mathrm{s}^{-1}$, for $5-10$ breaths, using feedback from a display of the transnasal pressure/flow plot on an oscilloscope (20 Mhz dual trace, V209-portable; Hitachi Australia, Sydney, Australia). A resting period of $2 \mathrm{~min}$ was allowed between each voluntary hyperpnoeic run.

Following the above measurements, the protocol was repeated $10 \mathrm{~min}$ after instillation of $0.2 \mathrm{mg}$ of the topical nasal decongestant oxymetazoline hydrochloride (Drixine $\mathbb{R}$, Schering-Plough, Auckland, New Zealand; $0.5 \mathrm{mg}$. $\mathrm{mL}^{-1}$ ) into each nostril using an atomizer (Devilbiss No. 15; Medical Gases Australia, Annandale, Australia). It was reasoned that administration of a topical nasal decongestant would minimize the vascular component of $R \mathrm{n}$ and thus increase the resolution of the study in terms of the effect of the ENDS on the underlying structural or anatomical determinants of $R$.

\section{Data analysis}

Nasal airflow dynamics were characterized using the power function analysis of JAEGER and MATTHYS [14] as modified by WHEATLEY et al. [11]. Consequently, a power function of the form $P=\mathrm{a} V^{\mathrm{b}}$ (where $P$ represents transnasal pressure, $V^{\top}$ represents nasal flow and a and $\mathrm{b}$ are constants) was fitted separately to both the inspiratory and expiratory transnasal pressure/flow curves by the method of least squares [10]. During tidal breathing, inspiratory and expiratory $R \mathrm{n}$ were calculated from the fitted power functions at a nasal airflow of $0.4 \mathrm{~L} \cdot \mathrm{s}^{-1}$.

During hyperpnoea, there was hysteresis of the inspiratory transnasal pressure/flow relationship. Thus there was no unique curve fit that adequately represented all of the inspiratory data. Consequently, inspiratory $R \mathrm{n}$ during hyperpnoea was measured directly from the raw data using the transnasal pressure (measured directly from the transnasal pressure/flow plots) at an inspiratory airflow of 1.0 $\mathrm{L} \cdot \mathrm{s}^{-1}$ (the peak flow achieved). In addition, the magnitude of the hysteresis of the inspiratory transnasal pressure/flow relationship was estimated as the difference between the transnasal pressures measured from the ascending and descending limbs of the inspiratory transnasal pressure/ flow plot at an inspiratory airflow of $0.5 \mathrm{~L} \cdot \mathrm{s}^{-1}$. No measurements were made during expiration in this phase of the study. 
For each condition in each subject, four or five inspiratory (and, for tidal breathing, four or five expiratory) transnasal pressure/flow plots were constructed, each from three to five consecutive and representative breaths. Values obtained from these plots were then averaged to give individual mean values for each subject for each condition. Individual values were then pooled and group mean results calculated.

Group results were compared using one-factor repeated measures analysis of variance with Fisher's protected least significant difference test used as a post hoc multiple comparison technique. Within each subject, a one-tailed unpaired t-test was used to compare control and ENDS values in order to determine those subjects in whom $R$ n significantly decreased with the ENDS (i.e. "responders"). The $R \mathrm{n}$ for the "responders" was compared to that of the remaining subjects (i.e. "nonresponders") using an unpaired t-test. The correlation between control levels of $R \mathrm{n}$ and the change in $R \mathrm{n}$ with the ENDS was examined using linear regression analysis. NPSs were compared using a Wilcoxon signed-rank test with Bonferroni correction for multiple comparisons, and the correlation between NPSs and the level of $R \mathrm{n}$ was examined using Spearman rank correlation. A p-value of $<0.05$ was considered significant.

\section{Results}

\section{Curve fitting}

The power function $P=\mathrm{a} V^{\mathrm{b}}$ fitted the inspiratory and expiratory transnasal pressure/flow data with an $r^{2}$ of 0.90 1.0 for all of the conditions studied.

\section{Tidal breathing}

Representative transnasal pressure/flow plots obtained in one subject during control and administration of placebo and the ENDS are shown in figure 1. Group mean inspiratory and expiratory a, inspiratory $b$ and inspiratory and expiratory $R \mathrm{n}\left(\right.$ at $0.4 \mathrm{~L} \cdot \mathrm{s}^{-1}$ ) were unaffected by pla- cebo but were significantly lower with the ENDS $(\mathrm{p}<$ 0.05; table 1; fig. 2).

When the data for each subject were examined separately, $R \mathrm{n}$ (at $0.4 \mathrm{~L} \cdot \mathrm{s}^{-1}$ ) decreased significantly in six subjects during both inspiration and expiration, in one subject during inspiration only and in two subjects during expiration only (fig. 2, table 2). Control inspiratory (but not expiratory) $R \mathrm{n}$ (at $0.4 \mathrm{~L} \cdot \mathrm{s}^{-1}$ ) was higher in "responders" $\left(3.28 \pm 0.16 \mathrm{cmH}_{2} \mathrm{O} \cdot \mathrm{L}^{-1} \cdot \mathrm{s}\right)$ than "nonresponders" $(2.60 \pm$ $\left.0.08 \mathrm{cmH}_{2} \mathrm{O} \cdot \mathrm{L}^{-1} \cdot \mathrm{s}, \mathrm{p}=0.04\right)$. In addition, there was a significant positive correlation between control $R \mathrm{n}$ (at 0.4 $\left.\mathrm{L} \cdot \mathrm{s}^{-1}\right)$ and the decrease in $R \mathrm{n}\left(\right.$ at $\left.0.4 \mathrm{~L} \cdot \mathrm{s}^{-1}\right)$ with the ENDS for both inspiration and expiration $(\mathrm{r}=0.58, \mathrm{p}=0.01$ and $\mathrm{r}=0.47, \mathrm{p}=0.04$, respectively).

\section{Effect of nasal decongestant during tidal breathing}

The group mean inspiratory and expiratory a and $R \mathrm{n}$ (at $0.4 \mathrm{~L} \cdot \mathrm{s}^{-1}$ ) (but not b) decreased with nasal decongestant and then decreased further with the ENDS but not placebo $(p<0.05$; table 1, fig. 3). Following nasal decongestant the number of "responders" more than doubled to 19 (15 during inspiration and expiration, two during inspiration only and two during expiration only; table 2).

When the effects of the ENDS and nasal decongestant were compared, it was found that the ENDS alone reduced the group mean inspiratory and expiratory $R \mathrm{n}\left(\right.$ at $\left.0.4 \mathrm{~L} \cdot \mathrm{s}^{-1}\right)$ by approximately 18 and $16 \%$ respectively, whereas there was a 46 and $47 \%$ decrease with nasal decongestant. However, since the ENDS also reduced $R \mathrm{n}$ (at $0.4 \mathrm{~L} \cdot \mathrm{s}^{-1}$ ) after decongestant, the combination of the ENDS and nasal decongestant reduced $R \mathrm{n}\left(\right.$ at $\left.0.4 \mathrm{~L} \cdot \mathrm{s}^{-1}\right)$ by approximately $63 \%$ during inspiration and $66 \%$ during expiration (i.e. the effects of nasal decongestant and ENDS were additive).

\section{Hyperpnoea}

Technically acceptable data were obtained in 17 subjects during hyperpnoea. Representative inspiratory transnasal pressure/flow plots obtained during voluntary hyperpnoea
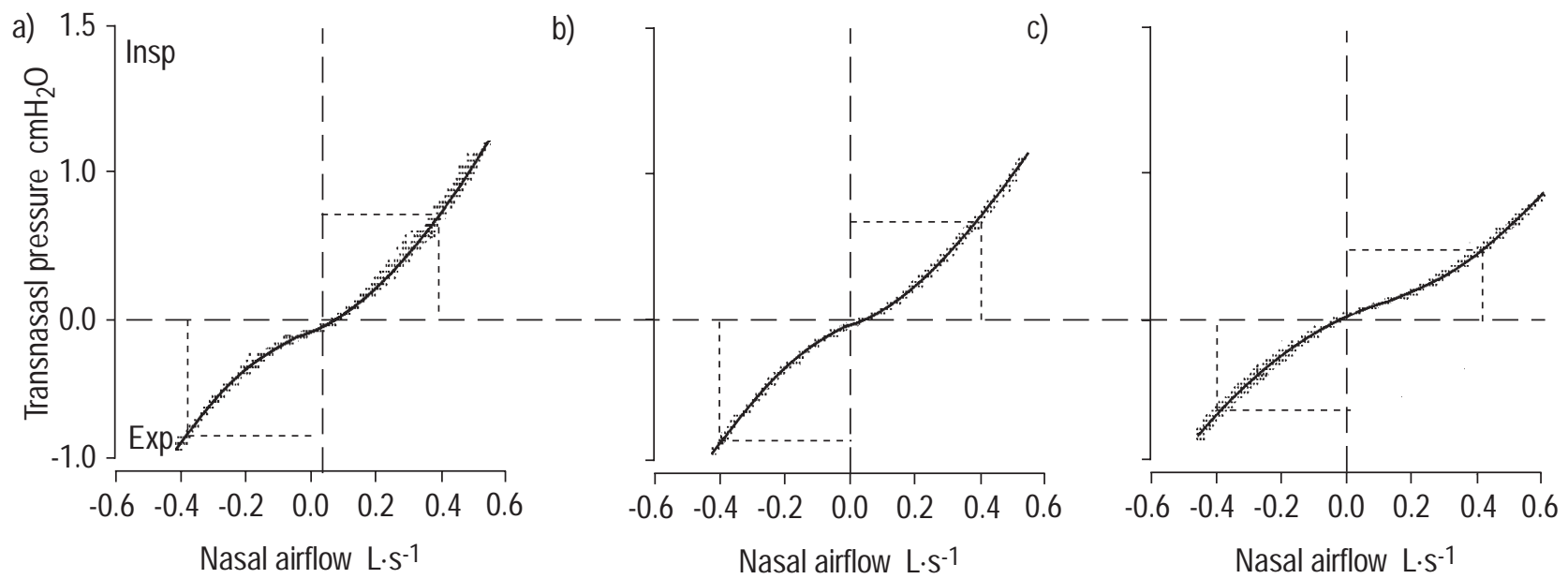

Fig. 1. - Representative transnasal pressure/flow plots obtained from five tidal breaths in one subject: a) without the external nasal dilator strip (ENDS) (control); b) with placebo; and c) with the ENDS. Individual data points are shown. inspiratory and expiratory nasal airflows of $0.4 \mathrm{~L} \cdot \mathrm{s}^{-1}$. Note the similar curves for control and placebo and the less steep relationship obtained with the ENDS. Insp: inspiration; Exp: expiration. 
Table 1. - Influence of the external nasal dilator strip (ENDS) on nasal airflow dynamics

\begin{tabular}{|c|c|c|c|c|c|c|}
\hline & \multicolumn{3}{|c|}{ Inspiration } & \multicolumn{3}{|c|}{ Expiration } \\
\hline & Control & Placebo & ENDS & Control & Placebo & ENDS \\
\hline \multicolumn{7}{|l|}{ Before nasal decongestant } \\
\hline a & $5.40 \pm 0.75$ & $5.48 \pm 0.69$ & $3.96 \pm 0.39^{\#}$ & $4.73 \pm 0.56$ & $5.05 \pm 0.67$ & $3.83 \pm 0.37^{\#}$ \\
\hline b & $1.79 \pm 0.03$ & $1.78 \pm 0.03$ & $1.71 \pm 0.02^{\#}$ & $1.68 \pm 0.03$ & $1.65 \pm 0.03$ & $1.65 \pm 0.03$ \\
\hline$R \mathrm{n}$ at $0.4 \mathrm{~L} \cdot \mathrm{s}^{-1} \mathrm{cmH}_{2} \mathrm{O} \cdot \mathrm{L}^{-1} \cdot \mathrm{s}$ & $2.52 \pm 0.28$ & $2.64 \pm 0.31$ & $2.07 \pm 0.20^{\#}$ & $2.51 \pm 0.26$ & $2.77 \pm 0.34$ & $2.12 \pm 0.20^{\#}$ \\
\hline$R \mathrm{n}$ at $1.0 \mathrm{~L} \cdot \mathrm{s}^{-1} \mathrm{cmH}_{2} \mathrm{O} \cdot \mathrm{L}^{-1} \cdot \mathrm{s}$ & $5.46 \pm 0.68$ & $5.73 \pm 0.77$ & $4.19 \pm 0.51^{\#}$ & - & - & - \\
\hline Hysteresis $\mathrm{cmH}_{2} \mathrm{O}$ & $0.89 \pm 0.15$ & $0.84 \pm 0.13$ & $0.54 \pm 0.09^{\#}$ & - & - & - \\
\hline \multicolumn{7}{|l|}{ After nasal decongestant } \\
\hline $\mathrm{a}$ & $2.70 \pm 0.25^{*}$ & $2.73 \pm 0.32 *$ & $1.86 \pm 0.13 * *$ & $2.63 \pm 0.27 *$ & $2.44 \pm 0.23 *$ & $1.72 \pm 0.12^{* \#}$ \\
\hline 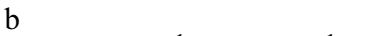 & $1.75 \pm 0.03$ & $1.76 \pm 0.02$ & $1.75 \pm 0.02$ & $1.71 \pm 0.03$ & $1.73 \pm 0.03$ & $1.77 \pm 0.05$ \\
\hline$R \mathrm{n}$ at $0.4 \mathrm{~L} \cdot \mathrm{s}^{-1} \mathrm{cmH}_{2} \mathrm{O} \cdot \mathrm{L}^{-1} \cdot \mathrm{s}$ & $1.37 \pm 0.13^{*}$ & $1.35 \pm 0.15^{*}$ & $0.93 \pm 0.06^{*}$ & $1.32 \pm 0.11 *$ & $1.25 \pm 0.12 *$ & $0.86 \pm 0.05^{* \#}$ \\
\hline$R \mathrm{n}$ at $1.0 \mathrm{~L} \cdot \mathrm{s}^{-1} \mathrm{cmH}_{2} \mathrm{O} \cdot \mathrm{L}^{-1} \cdot \mathrm{s}$ & $2.74 \pm 0.30^{*}$ & $3.23 \pm 0.61 *$ & $1.87 \pm 0.16^{* \#}$ & - & - & - \\
\hline Hysteresis $\mathrm{cmH}_{2} \mathrm{O}$ & $0.40 \pm 0.07 *$ & $0.60 \pm 0.16$ & $0.27 \pm 0.04^{*+}$ & - & - & - \\
\hline
\end{tabular}

Data are mean \pm SEM $(\mathrm{n}=20)$. a and $\mathrm{b}$ are obtained from the power function $P=\mathrm{a} V^{\mathrm{b}}$ (where $P$ represents transnasal pressure, $V^{\prime}$ represents nasal flow and $\mathrm{a}$ and $\mathrm{b}$ are constants. Nasal airflow resistance $(R \mathrm{n})$ was calculated at an airflow of $0.4 \mathrm{~L} \cdot \mathrm{s}^{-1}$ during tidal breathing and at an inspiratory airflow of $1.0 \mathrm{~L} \cdot \mathrm{s}^{-1}$ during voluntary hyperpnoea. The magnitude of the hysteresis of the inspiratory transnasal pressure/ flow relationship was estimated as the difference between the transnasal pressures measured from the ascending and descending inspiratory transnasal pressure/flow curve at a nasal airflow of $0.5 \mathrm{~L} \cdot \mathrm{s}^{-1}$ during voluntary hyperpnoea. ${ }^{\#}: \mathrm{p}<0.05$ compared with control and placebo; ${ }^{+}: \mathrm{p}<0.05$ compared with placebo; $*$ : $<<0.05$ compared with values obtained before use of nasal decongestant.

are shown in figure 4. For the group, inspiratory $R \mathrm{n}$ (at 1.0 $\left.\mathrm{L} \cdot \mathrm{s}^{-1}\right)$ was not significantly different between control and placebo but decreased with the ENDS ( $p<0.05$; table 1). During hyperpnoea there were eight inspiratory "responders" (table 2), seven of whom were the same subjects who responded to the ENDS during resting tidal breathing. Transnasal pressure/flow hysteresis was unaffected by placebo but was significantly reduced with the ENDS in nine subjects $(\mathrm{p}<0.05$, tables 1 and 2$)$.

\section{Effect of nasal decongestant during hyperpnoea}

For the group, inspiratory $R \mathrm{n}\left(\right.$ at $1.0 \mathrm{~L} \cdot \mathrm{s}^{-1}$ ) was significantly decreased with nasal decongestant and then reduced further in 15 subjects with the ENDS ( $p<0.05$; tables 1 and 2). Nasal decongestant decreased the inspiratory hysteresis of the transnasal pressure/flow curve for control and the ENDS $(\mathrm{p}<0.05)$, although not for placebo (table 1). Postdecongestant hysteresis with the ENDS was significantly less than that with placebo $(\mathrm{p}<0.05)$ but not significantly different from control (table 1). During voluntary hyperpnoea, the ENDS decreased predecongestant inspiratory $R \mathrm{n}\left(\right.$ at $1.0 \mathrm{~L} \cdot \mathrm{s}^{-1}$ ) by approximately $23 \%$, whereas nasal decongestant resulted in a $50 \%$ decrease. The combination of the ENDS and nasal decongestant resulted in a decrease in $R \mathrm{n}\left(\right.$ at $1.0 \mathrm{~L} \cdot \mathrm{s}^{-1}$ ) of approximately $66 \%$. In addition, ENDS decreased inspiratory hysteresis by approximately $39 \%$, whereas nasal decongestant resulted in a $55 \%$ decrease. The combination of ENDS and nasal decongestant resulted in a decrease in hysteresis of approximately $70 \%$.

\section{Perception of nasal blockage}

During tidal breathing the NPS was significantly reduced by the ENDS ( $p=0.001$; table 3 ). Eight of the nine subjects with lowered inspiratory and/or expiratory $R \mathrm{n}$ (at $0.4 \mathrm{~L} \cdot \mathrm{s}^{-1}$ ) with the ENDS also recorded a decrease in NPS with the ENDS. However, seven of the 11 "nonresponders" also reported a decrease in NPS. Administration of
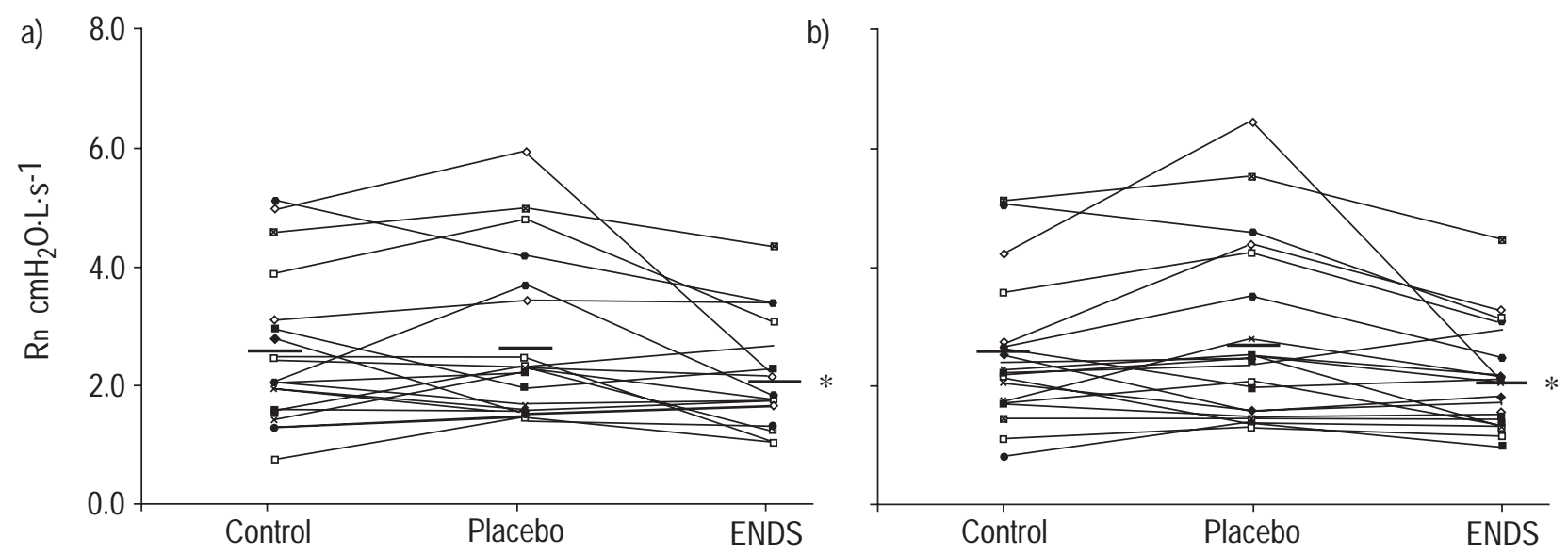

Fig. 2. - Individual data for: a) inspiratory; and b) expiratory nasal airflow resistance $(R \mathrm{n})\left(\right.$ at $\left.0.4 \mathrm{~L} \cdot \mathrm{s}^{-1}\right)$ under control, placebo and external nasal dilator strip (ENDS) conditions in 20 normal subjects during relaxed tidal breathing. Different symbols represent individual subjects and horizontal bars mean values. *: p<0.05 compared to both control and placebo. 
Table 2. - Number of subjects and direction of change in nasal airflow dynamics parameters with the external nasal dilator strip (ENDS)*

\begin{tabular}{|c|c|c|c|c|c|c|}
\hline & \multicolumn{6}{|c|}{$\mathrm{n}$} \\
\hline & \multicolumn{3}{|c|}{ Inspiration } & \multicolumn{3}{|c|}{ Expiration } \\
\hline & Decrease & No change & Increase & Decrease & No change & Increase \\
\hline \multicolumn{7}{|l|}{ Before decongestant } \\
\hline $\mathrm{a}$ & 7 & 11 & 2 & 8 & 10 & 2 \\
\hline b & 6 & 14 & 0 & 3 & 16 & 1 \\
\hline$R \mathrm{n}$ at $0.4 \mathrm{~L} \cdot \mathrm{s}^{-1} \mathrm{cmH}_{2} \mathrm{O} \cdot \mathrm{L}^{-1} \cdot \mathrm{s}$ & 7 & 9 & 4 & 8 & 9 & 3 \\
\hline$R \mathrm{n}$ at $1.0 \mathrm{~L} \cdot \mathrm{s}^{-1} \mathrm{cmH}_{2} \mathrm{O} \cdot \mathrm{L}^{-1} \cdot \mathrm{s}$ & 8 & 8 & 1 & - & - & - \\
\hline Hysteresis $\mathrm{cmH}_{2} \mathrm{O}$ & 9 & 7 & 1 & - & - & - \\
\hline \multicolumn{7}{|l|}{ After decongestant } \\
\hline $\mathrm{a}$ & 15 & 5 & 0 & 17 & 3 & 0 \\
\hline b & 3 & 14 & 3 & 1 & 17 & 2 \\
\hline$R$ at $0.4 \mathrm{~L} \cdot \mathrm{s}^{-1} \mathrm{cmH}_{2} \mathrm{O} \cdot \mathrm{L}^{-1} \cdot \mathrm{s}$ & 17 & 3 & 0 & 17 & 3 & 0 \\
\hline$R \mathrm{n}$ at $1.0 \mathrm{~L} \cdot \mathrm{s}^{-1} \mathrm{cmH}_{2} \mathrm{O} \cdot \mathrm{L}^{-1} \cdot \mathrm{s}$ & 15 & 2 & 0 & - & - & - \\
\hline Hysteresis $\mathrm{cmH}_{2} \mathrm{O}$ & 13 & 3 & 1 & - & - & - \\
\hline
\end{tabular}

*: compared with control $(\mathrm{p}<0.05)$. a and $\mathrm{b}$ are obtained from the power function $P=\mathrm{aV}^{\prime \mathrm{b}}$, where $P$ represents transnasal pressure, $V^{\prime}$ represents nasal flow and $\mathrm{a}$ and $\mathrm{b}$ are constants. Nasal airflow resistance $(R \mathrm{n})$ was calculated at an airflow of $0.4 \mathrm{~L} \cdot \mathrm{s}^{-1}$ during tidal breathing and at an inspiratory airflow of $1.0 \mathrm{~L} \cdot \mathrm{s}^{-1}$ during voluntary hyperpnoea. The magnitude of the hysteresis of the inspiratory transnasal pressure/flow relationship was estimated as the difference between the transnasal pressures measured from the ascending and descending inspiratory transnasal pressure/flow curves at a nasal airflow of $0.5 \mathrm{~L} \cdot \mathrm{s}^{-1}$ during voluntary hyperpnoea.

nasal decongestant resulted in a significant fall in NPS $(\mathrm{p}<0.05$; table 3$)$ with the addition of the ENDS resulting in a further fall $(\mathrm{p}<0.05$; table 3$)$. Ten of 19 postdecongestant inspiratory and/or expiratory "responders" reported a decrease in NPS with postdecongestant ENDS.

There was a significant correlation between the $R \mathrm{n}$ and the NPS across all conditions studied $(\mathrm{r}=0.746, \mathrm{p}<0.001)$. This relationship was stronger for the pre- and postdecongestant data $(\mathrm{r} s=0.809, \mathrm{p}<0.001)$ than for the pre- and postENDS data $(\mathrm{r} s=0.535, \mathrm{p}<0.001)$.

\section{Discussion}

Numerous mechanical devices for the dilation of the nostrils have been marketed over the years [15-17]. None, however, has gained widespread acceptance. The current
ENDS device attaches to the external surface of the nose and is held in place with adhesive tape. The lightweight nature, secure placement and ease of use of the ENDS have contributed to its popular acceptance. The ENDS device has been advocated for the relief of the symptoms of nasal obstruction associated with viral, allergic and pregnancy-related [18] nasal congestion, as well as symptoms related to a deviated nasal septum and collapsed nasal cartilages $[2,19]$. The device is also promoted as a method for the reduction of snoring [20-22]. In addition, the ENDS has become increasingly adopted by athletes to promote nasal breathing during exercise [23].

There have been previous studies examining the influence on $R \mathrm{n}$, in normal subjects, of both internal $[15,17$, $24,25]$ and external $[16,24,26]$ mechanical nasal dilators, including the ENDS [2, 6-9]. However, none of these previous studies are as comprehensive as the present study,
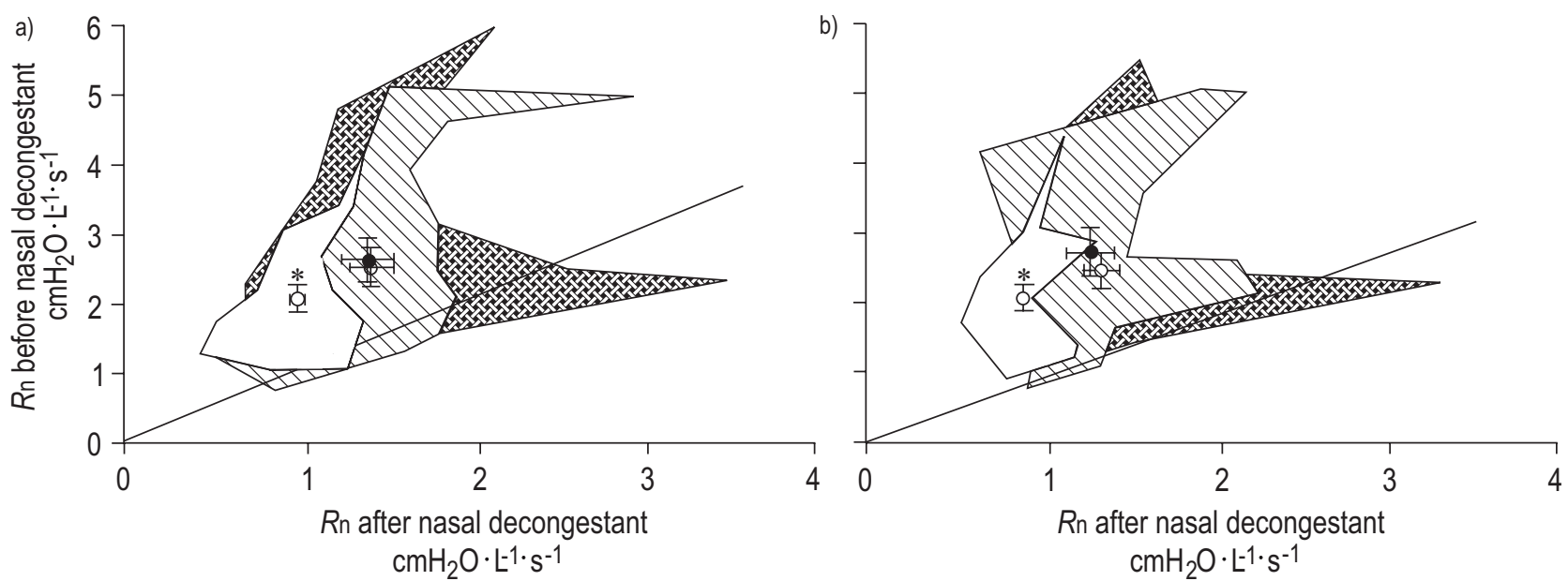

Fig. 3. - Identity plot showing group mean (circles) and range for nasal airflow resistance $(R \mathrm{n})\left(\right.$ at $\left.0.4 \mathrm{~L} \cdot \mathrm{s}^{-1}\right)$ before versus after use of nasal decongestant

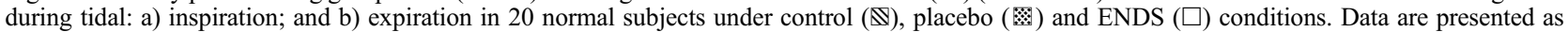
mean \pm SEM. Note that most values fall above the identity line $(-)$, indicating a decrease in $R$ n with nasal decongestant. However, the ENDS data are also significantly shifted both down and to the left (i.e. lower $R$ n compared to both placebo and control) both before and after use of nasal decongestant; *: $\mathrm{p}<0.05$ compared to control and placebo (both before and after use of decongestant). 


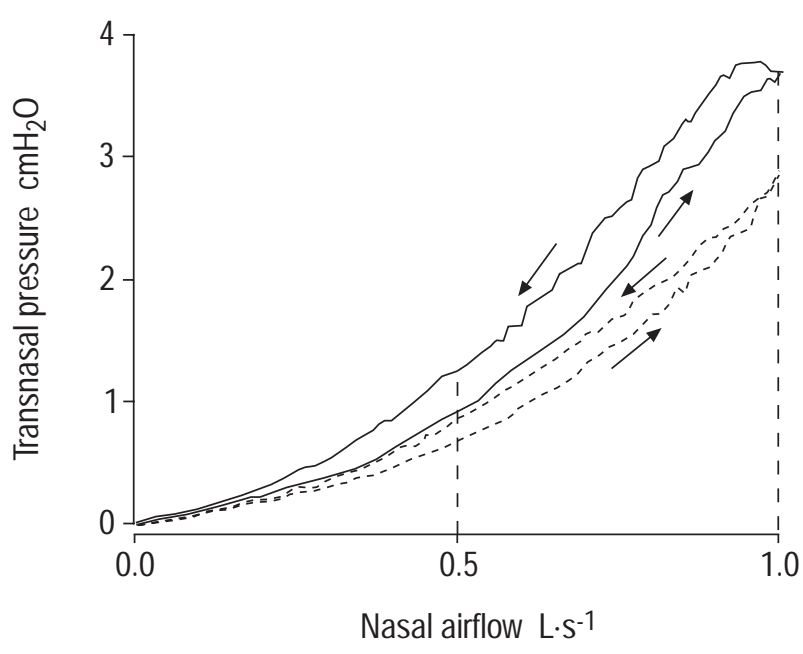

Fig. 4. - Representative mean inspiratory transnasal pressure/flow plots obtained from three breaths during voluntary hyperpnoea in one subject without (control; - - ) and with an external nasal dilator strip (ENDS; - - -). Note that with the ENDS there is a decrease in both nasal airflow resistance at $1.0 \mathrm{~L} \cdot \mathrm{s}^{-1}$ and hysteresis at $0.5 \mathrm{~L} \cdot \mathrm{s}^{-1}$. Arrows indicate direction of inspiratory transnasal pressure/flow hysteresis.

and none have examined the influence of the ENDS on nasal airflow regimens or expiratory $R \mathrm{n}$ or included comparisons with a placebo device. In addition, the concept of "responders" and "nonresponders" to the ENDS has not been examined in detail previously and there has been only one prior study that attempted to address factors that may determine "responsiveness" to the ENDS [10]. The present study is also the first to examine the effect of the ENDS on inspiratory $R \mathrm{n}$ and inspiratory transnasal pressure/flow hysteresis during hyperpnoea, the incidence of responders to the ENDS following nasal decongestant, and the relationship between individual perceptions of the influence of the ENDS on nasal patency and actual changes in $R$ n.

Using acoustic rhinometry, the ENDS device has been shown to increase the minimum cross-sectional area of the nasal passages (i.e. nasal valve) in normal subjects by some $9-43 \%[1,2,7,8]$. In a recent study, it was demonstrated that the dilatory effect of the ENDS is sustained over an 8-h period [10]. There is, however, some heterogeneity in the effect of the ENDS on nasal airway calibre. GRIFFEN et al. [1] demonstrated that the ENDS increased nasal valve cross-sectional area in normal subjects by an average of $0.30 \mathrm{~cm}^{2}$ or $25 \%$. However, some subjects experienced no increase in valve cross-sectional area $(23 \%$ of subjects had an increase of $\leq 10 \%$ ), whereas others experienced a

Table 3. - Influence of the external nasal dilator strip (ENDS) on nasal patency score (NPS)

\begin{tabular}{lccc}
\hline & \multicolumn{3}{c}{ NPS AU } \\
\cline { 2 - 4 } & Control & Placebo & ENDS \\
\hline $\begin{array}{c}\text { Before nasal } \\
\text { decongestant }\end{array}$ & $1.68 \pm 0.16$ & $1.53 \pm 0.16$ & $0.95 \pm 0.14^{\#}$ \\
$\begin{array}{c}\text { After nasal } \\
\text { decongestant }\end{array}$ & $0.53 \pm 0.08^{*}$ & $0.53 \pm 0.08^{*}$ & $0.23 \pm 0.07^{*}$ \\
\hline
\end{tabular}

Data are mean $\pm \operatorname{SEM}(n=20)$. AU: arbitrary unit; ${ }^{\#}: \mathrm{p}<0.05$ compared with control and placebo; *: $\mathrm{p}<0.05$ compared with after nasal decongestant. large increase (up to $185 \%$ ). This degree of between-subject variation in the magnitude of nasal valve dilation might be expected to influence the magnitude of any resulting changes in $R \mathrm{n}$. Indeed, some studies have shown significant falls in $R \mathrm{n}$ with the ENDS in normal subjects, ranging $14-30 \%[2,6-8]$ whereas other studies have failed to demonstrate any effect [9]. The present study has also shown that, although the ENDS reduced both the inspiratory and expiratory group mean $R \mathrm{n}$ by an average of 18 and $16 \%$ respectively, there was a large range in the magnitude of the response with some subjects failing to respond, or, even, showing an increased $R \mathrm{n}$ with the ENDS. These findings introduce the concept of some individuals responding to the device ("responders") while others do not ("nonresponders").

In the present study, the magnitude of the fall in inspiratory $R \mathrm{n}$ with the ENDS correlated positively with the magnitude of the control $R \mathrm{n}$. Thus the higher the resting $R \mathrm{n}$ the more likely it is that a given subject will respond to the ENDS with a substantial decrease in $R \mathrm{n}$. The corollary of this finding being that the ENDS is unlikely to lower $R \mathrm{n}$ much further in a subject whose resting $R \mathrm{n}$ is already at the lower end of the normal range. It has also been shown recently that in normal subjects there is a three-fold variation in the magnitude of lateral nasal vestibule wall compliance [10]. Consequently, another potential factor in determining ENDS "responsiveness" may lie in differences in the intrinsic elastic properties of the nasal vestibule walls, with "stiffer" noses dilating relatively less with the ENDS than more compliant noses. External nasal geometry (e.g. nasal width) may also play a role since this will influence the resultant force vector exerted by the ENDS device on the lateral nasal vestibule wall [10].

The a determined from the fitted power function is a measure of the tilt of the pressure/flow curve and, in the absence of substantial changes in $\mathrm{b}$, is representative of $R \mathrm{n}$ across all the flow rates studied $[11,14]$. Thus, the reduction in a with the ENDS is indicative of a fall in $R \mathrm{n}$ across all flow rates. To interpret the nature of the flow regimen, $b$ may be used [11]. Thus, the decrease in $b$ with the ENDS is indicative of a trend towards a less turbulent flow regimen with the ENDS in place. This may relate to alterations in the shape or size of the nasal valve region with the ENDS.

The effect of the ENDS on nasal airflow dynamics is attributable to the polyester springs embedded in the device since there was no significant effect of the placebo. However, the magnitude of this effect was less than that of decongestant. This contrasts with the report of RoITHMANN et al. [2] who found the ENDS to be equally as effective as decongestant at reducing $R \mathrm{n}$ in normal subjects. In the present study, the influence of the ENDS and decongestant were additive with combined reductions of 63 and $65 \%$ being achieved for inspiratory and expiratory $R \mathrm{n}$, respectively. Furthermore, after the application of decongestant, most subjects showed decreased inspiratory and expiratory $R \mathrm{n}$. If the effect of nasal decongestant is viewed as removing the vascular component of $R \mathrm{n}$, then the postdecongestant effect of the ENDS may provide a better estimate of the efficacy of the ENDS in reducing that part of the structural (or anatomical) component of $R \mathrm{n}$ which is determined by the lateral nasal vestibule walls. In addition, this finding suggests that some "nonresponders" (based on predecongestant data) show a reduction in the 
nasal valve-related structural component of their $R \mathrm{n}$ with the ENDS. However, in these subjects, either the magnitude of this component itself or the effect of the ENDS is so small that they cannot be separately distinguished from the much larger vascular component.

The use of the ENDS during exercise [23] raises the question of the influence of the ENDS on $R \mathrm{n}$ at high nasal airflow rates. In the present study, measurements were made of $R \mathrm{n}$ (at $1.0 \mathrm{~L} \cdot \mathrm{s}^{-1}$ ) during hyperpnoea to give an indication of the effect of the ENDS at high inspiratory flow rates. Compared to control and placebo, the ENDS reduced inspiratory $R \mathrm{n}\left(\right.$ at $\left.1.0 \mathrm{~L} \cdot \mathrm{s}^{-1}\right)$ during hyperpnoea. A characteristic feature of transnasal pressure/flow relationships during hyperpnoea is hysteresis of the inspiratory phase [27]. For the same inspiratory flow rate, transnasal pressures are higher during the latter part of inspiration (decreasing inspiratory flow rates) compared to earlier in inspiration (increasing inspiratory flow rates). SHI et al. [27] related inspiratory transnasal pressure/flow hysteresis to late inspiratory collapse of the lateral nasal vestibule wall that was, in turn, associated with the waning of alae nasi muscle activity towards the end of inspiration. In the present study, the ENDS, but not placebo, significantly reduced inspiratory transnasal pressure/flow hysteresis during hyperpnoea. This is similar to the effect of voluntary nasal flaring during hyperpnoea [27] and is most probably related to stabilization of the lateral nasal vestibule wall. Thus, the ENDS not only dilates the nasal airway (reducing $R \mathrm{n}$ and therefore the magnitude of intraluminal transnasal pressures) but may also stiffen the lateral nasal walls and oppose late-inspiratory collapsing forces (reducing hysteresis). The reduction in hysteresisrelated pressure losses during inspiration, together with the reduction in $R \mathrm{n}$, suggests that the ENDS may well reduce the inspiratory work of nasal breathing under conditions of high nasal airflow, such as might be encountered during exercise. Indeed, it has been recently demonstrated that during exercise the ENDS is associated with a decrease in the flow-resistive work of nasal breathing [28] and a delay in the onset of oral route breathing [29]. In the present study, however, during hyperpnoea the number of "responders" was not substantially increased, suggesting that subjects with a low $R$ n remain "nonresponders" during stimulated breathing.

In some previous studies, subjective assessments of nasal patency have been shown to correlate with quantitative measurements of $R \mathrm{n}$, although there can be considerable intersubject variability in the strength of this correlation $[30,31]$ with subjects having a higher $R \mathrm{n}$ being more accurate in their perception of nasal patency [3]. However, in other studies, subjective and objective evaluations of nasal patency have also been reported to differ [32]. In addition, since symptoms of nasal obstruction are typically associated with $R \mathrm{n}$ of $>3 \mathrm{cmH}_{2} \mathrm{O} \cdot \mathrm{L}^{-1} \cdot \mathrm{s}$ [33], it might have been expected that the normal subjects in the present study, most of whom had an inspiratory $R \mathrm{n}$ of $<3.0$ $\mathrm{cmH}_{2} \mathrm{O} \cdot \mathrm{L}^{-1} \cdot \mathrm{s}$ would have difficulty in detecting the $16-$ $18 \%$ decrease in $R \mathrm{n}$ which was associated with the ENDS. However, in contrast to the high degree of intersubject variability encountered in the magnitude and direction of the changes in $R \mathrm{n}$, the majority of subjects perceived an improvement in nasal patency with the ENDS. A major feature of the present findings, however, is that the majority of "nonresponders" also recorded a perception of improved nasal patency. Thus there were some individuals who perceived improved nasal patency when $R \mathrm{n}$ did not decrease. The perception of "less obstructed" nasal breathing appears to be a common finding in most normal subjects who use this device and may not be directly related to measures of $R$ n.

Thus, in most normal subjects, the external nasal dilator strip reduces nasal airflow resistance (especially if nasal airflow resistance is high) and also provides a sensation of improved nasal patency. Although it is not as effective in lowering nasal airflow resistance as topical nasal decongestant, these findings support the potential clinical application of the external nasal dilator strip as a nonpharmacological approach to the management of nasal obstruction, especially when nasal airflow resistance is above the normal range, and is associated with overt symptoms and when the obstruction is primarily associated with the nasal valve area. In support of this concept, a recent report [2] demonstrated an increase in nasal valve crosssectional area and a fall in nasal airflow resistance in patients with septal deviation, which was greater with the external nasal dilator strip than with nasal decongestion. In contrast, for patients with nasal congestion, topical nasal decongestant was more effective than the external nasal dilator strip in reducing nasal airflow resistance and relieving symptoms. However, since the effects of the external nasal dilator strip and topical nasal decongestant are additive, there may be additional benefits to be gained by combining the two approaches.

\section{References}

1. Griffen JW, Hunter G, Ferguson D, Sillers MJ. Physiologic effects of an external nasal dilator. Laryngoscope 1997; 107: 1235-1238.

2. Roithmann R, Chapnik J, Cole P, Szalai J, Zamel N. Role of the external nasal dilator in the management of nasal obstruction. Laryngoscope 1998; 108: 712-715.

3. Roithmann R, Chapnik J, Zamel N, Barreto SM, Cole P. Acoustic rhinometric assessment of the nasal valve. Am J Rhinol 1997; 11: 387-393.

4. Haight JSH, Cole P. The site and function of the nasal valve. Laryngoscope 1983; 93: 49-55.

5. Saunders MW, Jones NS, Kabala J, Lowe J. An anatomical, histological and magnetic resonance imaging study of the nasal septum. Clin Otolaryngol 1995; 20: 434-438.

6. Havas TE, Cochineas $\mathrm{C}$, Rhodes L. Is there a rationale for using nasal splints to enhance exercise performance? Mod Med Australia 1997; May: 130-132.

7. Gosepath J, Mann WJ, Amedee RG. Effects of the Breathe Right nasal strips on nasal ventilation. Am J Rhinol 1997; 11: 399-402.

8. Portugal LG, Mehta RH, Smith BE, Sabnani JB, Matava MJ. Objective assessment of the Breathe-Right device during exercise in adult males. Am J Rhinol 1997; 11: 393-397.

9. Vermoen CJ, Verbraak AFM, Bogarrd JM. Effect of a nasal dilatator on nasal patency during normal and forced nasal breathing. Int J Sports Med 1997; 19: 109-113.

10. Amis TC, Kirkness JP, Di Somma E, Wheatley JR. Nasal vestibule wall elasticity: interactions with a nasal dilator strip. J Appl Physiol 1999; 86: 1638-1643.

11. Wheatley JR, Amis TC, Engel LA. Nasal and oral pressure-flow relationships. J Appl Physiol 1991; 71: $2317-$ 2324. 
12. Kortekangas AK. Significance of anterior and posterior technique in rhinomanometry. Acta Otolaryngol 1972; 73: 218-221.

13. Schumacher MJ. Rhinomanometry. J Allergy Clin Immunol 1989; 83: 711-719.

14. Jaeger MJ, Matthys $H$. The pattern of flow in the upper human airways. Respir Physiol 1968; 6: 113-127.

15. Chuadry MR, Akhtar S, Dwalsaint F. Rhinomanometric evaluation of the improved mechanical therapeutic nasal dilator in patients with nasal obstruction. Rhinology 1996; 34: $32-34$

16. Ford CN, Rezakhany S, Ewanowski S. A nasal prosthesis for treatment of nasal airway obstruction. Rhinology 1985; 23: 223-229.

17. Petruson B. Improvement of the nasal airflow by the nasal dilator Nozovent. Rhinology 1988; 26: 289-292.

18. Turnbull GL, Rundell OH, Rayburn WF, Jones RK, Pearman CS. Managing pregnancy- related nocturnal nasal congestion: the external nasal dilator. J Reprod Med 1996; 41: 897-902.

19. Roithmann R, Cole P, Chapnik J, Shpirer I, Hoffstein V, Zamel N. Acoustic rhinometry in the evaluation of nasal obstruction. Laryngoscope 1995; 105: 275-281.

20. Scharf MB, McDannold MD, Zaretsky NT, Hux GT, Brannen DE, Berkowitz DV. Cyclic alternating pattern sequences in non-apneic snorers with and without nasal dilation. Ear Nose Throat $J$ 1996; 75: 617-619.

21. Scharf MB, Brannen DE, McDannold M. A subjective evaluation of a nasal dilator on sleep and snoring. Ear Nose Throat $J$ 1994; 73: 395-401.

22. Ulfberg J, Fenton G. Effect of Breathe Right nasal strip on snoring. Rhinology 1997; 35: 50-52.

23. Trocchio M, Wimmer JW, Parkman AW, Fisher J. Oxy- gen and exercise performance enhancing effects attributed to the Breathe Right $\AA$ nasal dilator. $J$ Athletic Training 1995; 30: 211-214.

24. Lorino A, Lofaso F, Drogou I, et al. Effects of different mechanical treatments on nasal resistance assessed by rhinometry. Chest 1998; 114: 166-170.

25. Hoffstein V, Mateika S, Metes A. Effect of nasal dilation on snoring and apneas during different stages of sleep. Sleep 1993; 16: 360-365.

26. Nielsen H, Orntoft S, Nielsen TG. Change in minimal cross sectional area using the new nasal dilator, "Airplus". Acta Otolaryngol Suppl (Stockh) 1997; 529: 130-132.

27. Shi Y, Seto-Poon M, Wheatley JR. Hysteresis of the nasal pressure-flow relationship during hyperpnea in normal subjects. J Appl Physiol 1998; 85: 286-293.

28. Garlick SR, Gehring JM, Wheatley JR, Amis TC. Nasal airflow resistance and the flow resistive work of nasal breathing during exercise: effects of a nasal dilator strip. Am J Respir Crit Care Med 1999; 159: A417.

29. Seto-Poon M, Amis TC, Kirkness J, Wheatley JR. Nasal dilator strips delay the onset of oro-nasal breathing during exercise. Can J Appl Physiol, 1999; 24: 538-547.

30. Simola M, Malmberg H. Sensation of nasal airflow compared with nasal airway resistance in patients with rhinitis. Clin Otolaryngol 1997; 22: 260-262.

31. Sipila J, Suonpaa J, Siloniemi P, Laippala P. Correlations between subjective sensation of nasal patency and rhinomanometry in both unilateral and total nasal assessment. $J$ Otorhinolaryngol Relat Spec 1995; 57: 260-263.

32. Eccles R, Jones AS. The effect of menthol on nasal resistance to air flow. J Laryngol Otol 1983; 97: 705-709.

33. McCaffrey TV, Kern EB. Clinical evaluation of nasal obstruction. Arch Otolaryngol 1979; 105: 542-545. 